Publications of the Astronomical Society of the Pacific, 114:708-720, 2002 July

(C) 2002. The Astronomical Society of the Pacific. All rights reserved. Printed in U.S.A.

\title{
The $Y$ Band at 1.035 Microns: Photometric Calibration and the Dwarf Stellar/Substellar Color Sequence
}

\author{
Lynne A. Hillenbrand and Jonathan B. Foster \\ California Institute of Technology, Department of Astronomy, MS 105-24, Pasadena, CA 91125; 1ah@astro.caltech.edu
}

S. E. PERsSON

Observatories of the Carnegie Institution of Washington, 813 Santa Barbara Street, Pasadena, CA 91101

AND

K. MatThews

California Institute of Technology, Palomar Observatory, MS 320-47, Pasadena, CA 91125

Received 2002 March 19; accepted 2002 April 10; published 2002 June 4

\begin{abstract}
We define and characterize a photometric bandpass (called " $Y$ ") that is centered near $1.035 \mu \mathrm{m}$, in between the traditionally classified "optical" and "infrared" spectral regimes. We present $Y$ magnitudes and $Y-H$ and $Y-K$ colors for a sample consisting mostly of photometric and spectral standards, spanning the spectral type range sdO to T5 V. Deep molecular absorption features in the near-infrared spectra of extremely cool objects are such that the $Y-H$ and $Y-K$ colors grow rapidly with advancing spectral type especially from late $\mathrm{M}$ through mid-L, substantially more rapidly than $J-H$ or $H-K$, which span a smaller total dynamic range. Consistent with other near-infrared colors, however, $Y-H$ and $Y-K$ colors turn blueward in the L6-L8 temperature range, with later T-type objects having colors similar to those of warmer $\mathrm{M}$ and $\mathrm{L}$ stars. $Y-J$ colors remain constant at $1.0 \pm 0.15 \mathrm{mag}$ from early-L through late-T dwarfs. The slope of the interstellar reddening vector within this filter is $A_{Y}=0.38 A_{V}$. Reddening moves stars nearly along the $Y H K$ dwarf color sequence, making it more difficult to distinguish unambiguously very low mass candidate brown dwarf objects from higher mass stars seen, e.g., through the Galactic plane or toward star-forming regions. Other diagrams involving the $Y$ band may be somewhat more discriminating.
\end{abstract}

\section{INTRODUCTION}

The traditional near-infrared photometric bandpasses that are observable from the ground through windows of moderate atmospheric transparency are $J H K L M N Q$ (and their slight variants $K^{\prime}, K_{s}, L^{\prime}, M^{\prime}, N 1, N 2, N 3$, etc.). This set of filters, defined broadly as in Johnson (1966), with the $H$ band being devised soon thereafter by Becklin and similarly the $Q$ band by Low, neglected to include one rather clean window through the atmosphere near $1.035 \mu \mathrm{m}$. This region of the electromagnetic spectrum is in between the traditional "optical" and "infrared" wavelength regimes, which are usually divided from one another based on detector technology (nowadays CCDs vs. $\mathrm{InSb}$ or $\mathrm{HgCdTe}$ arrays) around $1 \mu \mathrm{m}$. We discuss in this paper a filter called " $Y$ " that takes advantage of this window and holds particular interest for studies of low-mass and extremely cool stars and substellar objects, i.e., brown dwarfs.

The $Y$ filter bandpass was designed by S. E. P. and K. M. after visually inspecting the US Air Force's LOWTRAN (Selby \& McClatchey 1975) atmospheric transmission curve and also referring to Manducca \& Bell (1979). The half-power points of the filter were aimed at the $75 \%$ transmission points of the broad atmospheric absorption features near 0.955 and $1.112 \mu \mathrm{m}$. The filters were manufactured by Barr Associates (Worchester, MA). We name the filter " $Y$ " in publication as a compromise between its current discrepant designations ( $X$ and $Z$, as described below). Further, we choose $Y$ to distinguish this filter from $z$ and $Z$ filters in use, which are quantitatively different.

Nearly identical $Y$ filters reside in the Keck Near-Infrared Camera (NIRC; where it is called " $Z$ ") and the Palomar 60 inch cassegrain Infrared Camera (P60/IRC; where it is called " $X$ "), as well as in the Palomar 200 inch D-78 and D-80 cameras.

At $77 \mathrm{~K}$, the Keck/NIRC filter has $\lambda_{c}=1.032 \mu \mathrm{m}$ and $\delta \lambda_{c}=0.157 \mu \mathrm{m}$ with greater than $50 \%$ transmission from 0.9645 to $1.0950 \mu \mathrm{m}$, while the P60/IRC filter has $\lambda_{c}=1.04$ $\mu \mathrm{m}$ and $\delta \lambda_{c}=0.15 \mu \mathrm{m}$ with greater than $50 \%$ transmission from 0.9580 to $1.1080 \mu \mathrm{m}$. The two filter profiles are nearly identical in shape, with the Keck/NIRC filter 3\%-5\% less transmissive. It was found to be difficult to produce the specified filter with peak transmission much above $70 \%$; transmission profiles are given in Table 1. In Figure 1, filter transmission curves for the P60/IRC filter set are shown relative to a model of the atmo- 
TABLE 1

$Y$-Band Filter Transmission

\begin{tabular}{|c|c|c|c|c|c|c|c|c|}
\hline $\begin{array}{l}\text { Wavelength } \\
\text { (§) }\end{array}$ & $\begin{array}{c}\text { Transmission } \\
\text { (P60/IRC) }\end{array}$ & $\begin{array}{l}\text { Transmission } \\
\text { (Keck/NIRC) }\end{array}$ & $\begin{array}{l}\text { Wavelength } \\
(\AA)\end{array}$ & $\begin{array}{c}\text { Transmission } \\
\text { (P60/IRC) }\end{array}$ & $\begin{array}{l}\text { Transmission } \\
\text { (Keck/NIRC) }\end{array}$ & $\begin{array}{l}\text { Wavelength } \\
(\AA)\end{array}$ & $\begin{array}{c}\text { Transmission } \\
\text { (P60/IRC) }\end{array}$ & $\begin{array}{l}\text { Transmission } \\
\text { (Keck/NIRC) }\end{array}$ \\
\hline 9260 & 0.0000 & 0.0000 & $10000 \ldots \ldots$ & 0.7183 & 0.6708 & $10740 \ldots \ldots$ & 0.6200 & 0.5798 \\
\hline $9270 \ldots \ldots$ & 0.0016 & 0.0000 & $10010 \ldots \ldots$ & 0.7170 & 0.6655 & $10750 \ldots \ldots$ & 0.6178 & 0.5757 \\
\hline $9280 \ldots \ldots$ & 0.0032 & 0.0000 & $10020 \ldots \ldots$ & 0.7150 & 0.6628 & $10760 \ldots \ldots$ & 0.6154 & 0.5744 \\
\hline $9290 \ldots \ldots$ & 0.0044 & 0.0000 & $10030 \ldots \ldots$ & 0.7119 & 0.6614 & $10770 \ldots \ldots$ & 0.6129 & 0.5690 \\
\hline 9300 & 0.0050 & 0.0000 & 10040 & 0.7081 & 0.6574 & 10780 & 0.6100 & 0.5636 \\
\hline $9310 \ldots \ldots$ & 0.0060 & 0.0000 & $10050 \ldots \ldots$ & 0.7039 & 0.6547 & $10790 \ldots \ldots$ & 0.6068 & 0.5623 \\
\hline $9320 \ldots \ldots$ & 0.0070 & 0.0010 & $10060 \ldots \ldots$ & 0.7000 & 0.6534 & $10800 \ldots \ldots$ & 0.6032 & 0.5582 \\
\hline $9330 \ldots \ldots$ & 0.0080 & 0.0014 & $10070 \ldots \ldots$ & 0.6966 & 0.6534 & $10810 \ldots \ldots$ & 0.5993 & 0.5569 \\
\hline $9340 \ldots \ldots$ & 0.0100 & 0.0024 & $10080 \ldots \ldots$ & 0.6939 & 0.6493 & $10820 \ldots \ldots$ & 0.5950 & 0.5542 \\
\hline $9350 \ldots \ldots$ & 0.0130 & 0.0026 & $10090 \ldots \ldots$ & 0.6917 & 0.6480 & $10830 \ldots \ldots$ & 0.5905 & 0.5529 \\
\hline $9360 \ldots \ldots$ & 0.0160 & 0.0037 & $10100 \ldots \ldots$ & 0.6900 & 0.6467 & $10840 \ldots \ldots$ & 0.5861 & 0.5435 \\
\hline $9370 \ldots \ldots$ & 0.0180 & 0.0051 & $10110 \ldots \ldots$ & 0.6888 & 0.6426 & 10850 & 0.5825 & 0.5408 \\
\hline $9380 \ldots \ldots$ & 0.0200 & 0.0059 & $10120 \ldots \ldots$ & 0.6878 & 0.6426 & $10860 \ldots \ldots$ & 0.5800 & 0.5408 \\
\hline $9390 \ldots \ldots$ & 0.0250 & 0.0078 & $10130 \ldots \ldots$ & 0.6866 & 0.6426 & $10870 \ldots \ldots$ & 0.5790 & 0.5367 \\
\hline $9400 \ldots \ldots$ & 0.0300 & 0.0097 & $10140 \ldots \ldots$ & 0.6850 & 0.6427 & $10880 \ldots \ldots$ & 0.5785 & 0.5341 \\
\hline $9410 \ldots \ldots$ & 0.0350 & 0.0117 & $10150 \ldots \ldots$ & 0.6827 & 0.6427 & $10890 \ldots \ldots$ & 0.5775 & 0.5260 \\
\hline $9420 \ldots \ldots$ & 0.0400 & 0.0140 & $10160 \ldots \ldots$ & 0.6801 & 0.6427 & $10900 \ldots \ldots$ & 0.5750 & 0.5233 \\
\hline $9430 \ldots \ldots$ & 0.0500 & 0.0170 & $10170 \ldots \ldots$ & 0.6774 & 0.6373 & $10910 \ldots \ldots$ & 0.5701 & 0.5193 \\
\hline $9440 \ldots \ldots$ & 0.0600 & 0.0211 & $10180 \ldots \ldots$ & 0.6750 & 0.6359 & $10920 \ldots \ldots$ & 0.5636 & 0.5166 \\
\hline $9450 \ldots \ldots$ & 0.0700 & 0.0259 & $10190 \ldots \ldots$ & 0.6732 & 0.6319 & $10930 \ldots \ldots$ & 0.5565 & 0.5139 \\
\hline 9460 & 0.0800 & 0.0300 & $10200 \ldots \ldots$ & 0.6719 & 0.6292 & 10940 & 0.5500 & 0.5072 \\
\hline $9470 \ldots \ldots$ & 0.1100 & 0.0365 & $10210 \ldots \ldots$ & 0.6709 & 0.6279 & $10950 \ldots \ldots$ & 0.5448 & 0.5004 \\
\hline $9480 \ldots \ldots$ & 0.1300 & 0.0462 & $10220 \ldots \ldots$ & 0.6700 & 0.6265 & $10960 \ldots \ldots$ & 0.5408 & 0.4991 \\
\hline $9490 \ldots \ldots$ & 0.1633 & 0.0542 & $10230 \ldots \ldots$ & 0.6690 & 0.6239 & $10970 \ldots \ldots$ & 0.5377 & 0.4924 \\
\hline $9500 \ldots \ldots$ & 0.2000 & 0.0618 & $10240 \ldots \ldots$ & 0.6678 & 0.6225 & $10980 \ldots \ldots$ & 0.5350 & 0.4910 \\
\hline $9510 \ldots \ldots$ & 0.2315 & 0.0758 & $10250 \ldots \ldots$ & 0.6665 & 0.6225 & $10990 \ldots \ldots$ & 0.5326 & 0.4870 \\
\hline $9520 \ldots \ldots$ & 0.2530 & 0.0847 & $10260 \ldots \ldots$ & 0.6650 & 0.6225 & $11000 \ldots \ldots$ & 0.5306 & 0.4843 \\
\hline $9530 \ldots \ldots$ & 0.2730 & 0.1082 & $10270 \ldots \ldots$ & 0.6634 & 0.6212 & $11010 \ldots \ldots$ & 0.5296 & 0.4803 \\
\hline$\ldots \ldots$ & 0.3000 & 0.1264 & $10280 \ldots \ldots$ & 0.6619 & 0.6198 & $11020 \ldots \ldots$ & 0.5300 & 0.4776 \\
\hline $9550 \ldots \ldots$ & 0.3401 & 0.1505 & $10290 \ldots \ldots$ & 0.6607 & 0.6172 & $11030 \ldots \ldots$ & 0.5318 & 0.4736 \\
\hline $9560 \ldots \ldots$ & 0.3903 & 0.1716 & $10300 \ldots \ldots$ & 0.6600 & 0.6172 & $11040 \ldots \ldots$ & 0.5335 & 0.4736 \\
\hline $9570 \ldots \ldots$ & 0.4454 & 0.1944 & $10310 \ldots \ldots$ & 0.6599 & 0.6145 & $11050 \ldots \ldots$ & 0.5335 & 0.4695 \\
\hline $9580 \ldots \ldots$ & 0.5000 & 0.2192 & $10320 \ldots \ldots$ & 0.6604 & 0.6131 & $11060 \ldots \ldots$ & 0.5300 & 0.4668 \\
\hline $9590 \ldots \ldots$ & 0.5497 & 0.2539 & $10330 \ldots \ldots$ & 0.6612 & 0.6105 & $11070 \ldots \ldots$ & 0.5213 & 0.4669 \\
\hline $9600 \ldots \ldots$ & 0.5933 & 0.2948 & $10340 \ldots \ldots$ & 0.6620 & 0.6105 & $11080 \ldots \ldots$ & 0.5059 & 0.4669 \\
\hline $9610 \ldots \ldots$ & 0.6302 & 0.3248 & $10350 \ldots \ldots$ & 0.6628 & 0.6091 & $11090 \ldots \ldots$ & 0.4826 & 0.4642 \\
\hline $9620 \ldots \ldots$ & 0.6600 & 0.3938 & $10360 \ldots \ldots$ & 0.6635 & 0.6091 & $11100 \ldots \ldots$ & 0.4500 & 0.4628 \\
\hline $9630 \ldots \ldots$ & 0.6826 & 0.4533 & $10370 \ldots \ldots$ & 0.6642 & 0.6078 & $11110 \ldots \ldots$ & 0.4075 & 0.4588 \\
\hline $9640 \ldots \ldots$ & 0.6991 & 0.4982 & $10380 \ldots \ldots$ & 0.6650 & 0.6078 & $11120 \ldots \ldots$ & 0.3577 & 0.4480 \\
\hline $9650 \ldots \ldots$ & 0.7111 & 0.5455 & $10390 \ldots \ldots$ & 0.6659 & 0.6092 & $11130 \ldots \ldots$ & 0.3041 & 0.4426 \\
\hline $9660 \ldots \ldots$ & 0.7200 & 0.5871 & $10400 \ldots \ldots$ & 0.6671 & 0.6092 & $11140 \ldots \ldots$ & 0.2500 & 0.4292 \\
\hline $9670 \ldots \ldots$ & 0.7271 & 0.6177 & $10410 \ldots \ldots$ & 0.6684 & 0.6078 & $11150 \ldots \ldots$ & 0.1986 & 0.3942 \\
\hline $9680 \ldots \ldots$ & 0.7328 & 0.6435 & $10420 \ldots \ldots$ & 0.6700 & 0.6092 & $11160 \ldots \ldots$ & 0.1519 & 0.3713 \\
\hline $9690 \ldots \ldots$ & 0.7371 & 0.6621 & $10430 \ldots \ldots$ & 0.6718 & 0.6092 & $11170 \ldots \ldots$ & 0.1118 & 0.3349 \\
\hline 9700 & 0.7400 & 0.6748 & 10440 & 0.6735 & 0.6092 & $11180 \ldots \ldots$ & 0.0800 & 0.3147 \\
\hline $9710 \ldots \ldots$ & 0.7417 & 0.6864 & $10450 \ldots \ldots$ & 0.6747 & 0.6092 & $11190 \ldots \ldots$ & 0.0578 & 0.3038 \\
\hline $9720 \ldots \ldots$ & 0.7427 & 0.6976 & $10460 \ldots \ldots$ & 0.6750 & 0.6105 & $11200 \ldots \ldots$ & 0.0435 & 0.2929 \\
\hline $9730 \ldots \ldots$ & 0.7436 & 0.6999 & $10470 \ldots \ldots$ & 0.6743 & 0.6119 & $11210 \ldots \ldots$ & 0.0350 & 0.2819 \\
\hline $9740 \ldots \ldots$ & 0.7450 & 0.7057 & $10480 \ldots \ldots$ & 0.6728 & 0.6119 & $11220 \ldots \ldots$ & 0.0300 & 0.2710 \\
\hline $9750 \ldots \ldots$ & 0.7472 & 0.7090 & $10490 \ldots \ldots$ & 0.6712 & 0.6146 & $11230 \ldots \ldots$ & 0.0266 & 0.2601 \\
\hline $9760 \ldots \ldots$ & 0.7500 & 0.7109 & $10500 \ldots \ldots$ & 0.6700 & 0.6146 & $11240 \ldots \ldots$ & 0.0242 & 0.2492 \\
\hline $9770 \ldots \ldots$ & 0.7528 & 0.7124 & $10510 \ldots \ldots$ & 0.6696 & 0.6146 & $11250 \ldots \ldots$ & 0.0221 & 0.2383 \\
\hline $9780 \ldots \ldots$ & 0.7550 & 0.7136 & $10520 \ldots \ldots$ & 0.6697 & 0.6133 & $11260 \ldots \ldots$ & 0.0200 & 0.2274 \\
\hline $9790 \ldots \ldots$ & 0.7563 & 0.7151 & $10530 \ldots \ldots$ & 0.6699 & 0.6133 & $11270 \ldots \ldots$ & 0.0176 & 0.2164 \\
\hline $9800 \ldots \ldots$ & 0.7566 & 0.7152 & $10540 \ldots \ldots$ & 0.6700 & 0.6146 & $11280 \ldots \ldots$ & 0.0149 & 0.2055 \\
\hline $9810 \ldots \ldots$ & 0.7561 & 0.7165 & $10550 \ldots \ldots$ & 0.6695 & 0.6146 & $11290 \ldots \ldots$ & 0.0123 & 0.1946 \\
\hline $9820 \ldots \ldots$ & 0.7550 & 0.7165 & $10560 \ldots \ldots$ & 0.6685 & 0.6133 & $11300 \ldots \ldots$ & 0.0100 & 0.1837 \\
\hline $9830 \ldots \ldots$ & 0.7533 & 0.7165 & $10570 \ldots \ldots$ & 0.6670 & 0.6133 & $11310 \ldots \ldots$ & 0.0082 & 0.1728 \\
\hline $9840 \ldots \ldots$ & 0.7511 & 0.7165 & $10580 \ldots \ldots$ & 0.6650 & 0.6120 & $11320 \ldots \ldots$ & 0.0069 & 0.1618 \\
\hline $9850 \ldots \ldots$ & 0.7484 & 0.7165 & $10590 \ldots \ldots$ & 0.6627 & 0.6093 & $11330 \ldots \ldots$ & 0.0059 & 0.1509 \\
\hline $9860 \ldots \ldots$ & 0.7450 & 0.7134 & $10600 \ldots \ldots$ & 0.6601 & 0.6079 & $11340 \ldots \ldots$ & 0.0050 & 0.1400 \\
\hline $9870 \ldots \ldots$ & 0.7411 & 0.7125 & $10610 \ldots \ldots$ & 0.6575 & 0.6066 & $11350 \ldots \ldots$ & 0.0042 & 0.1291 \\
\hline $9880 \ldots \ldots$ & 0.7369 & 0.7110 & $10620 \ldots \ldots$ & 0.6550 & 0.6026 & $11360 \ldots \ldots$ & 0.0034 & 0.1182 \\
\hline $9890 \ldots \ldots$ & 0.7331 & 0.7083 & $10630 \ldots \ldots$ & 0.6527 & 0.6026 & $11370 \ldots \ldots$ & 0.0027 & 0.1072 \\
\hline $9900 \ldots \ldots$ & 0.7300 & 0.7058 & $10640 \ldots \ldots$ & 0.6504 & 0.5999 & $11380 \ldots \ldots$ & 0.0020 & 0.0963 \\
\hline $9910 \ldots \ldots$ & 0.7280 & 0.7019 & $10650 \ldots \ldots$ & 0.6479 & 0.5972 & $11390 \ldots \ldots$ & 0.0014 & 0.0854 \\
\hline $9920 \ldots \ldots$ & 0.7267 & 0.6993 & $10660 \ldots \ldots$ & 0.6450 & 0.5959 & $11400 \ldots \ldots$ & 0.0008 & 0.0745 \\
\hline $9930 \ldots \ldots$ & 0.7259 & 0.6964 & $10670 \ldots \ldots$ & 0.6415 & 0.5945 & $11410 \ldots \ldots$ & 0.0004 & 0.0636 \\
\hline $9940 \ldots \ldots$ & 0.7250 & 0.6935 & $10680 \ldots \ldots$ & 0.6375 & 0.5945 & $11420 \ldots \ldots$ & 0.0000 & 0.0527 \\
\hline $9950 \ldots \ldots$ & 0.7238 & 0.6891 & $10690 \ldots \ldots$ & 0.6336 & 0.5892 & $11430 \ldots \ldots$ & 0.0000 & 0.0417 \\
\hline $9960 \ldots \ldots$ & 0.7225 & 0.6841 & $10700 \ldots \ldots$ & 0.6300 & 0.5865 & $11440 \ldots \ldots$ & 0.0000 & 0.0308 \\
\hline $9970 \ldots \ldots$ & 0.7212 & 0.6816 & $10710 \ldots \ldots$ & 0.6270 & 0.5865 & $11450 \ldots \ldots$ & 0.0000 & 0.0199 \\
\hline $9980 \ldots \ldots$ & 0.7200 & 0.6770 & $10720 \ldots \ldots$ & 0.6244 & 0.5865 & $11460 \ldots \ldots$ & 0.0000 & 0.0090 \\
\hline $9990 \ldots \ldots$ & 0.7191 & 0.6739 & $10730 \ldots \ldots$ & 0.6222 & 0.5838 & $11470 \ldots \ldots$ & 0.0000 & 0.0000 \\
\hline
\end{tabular}




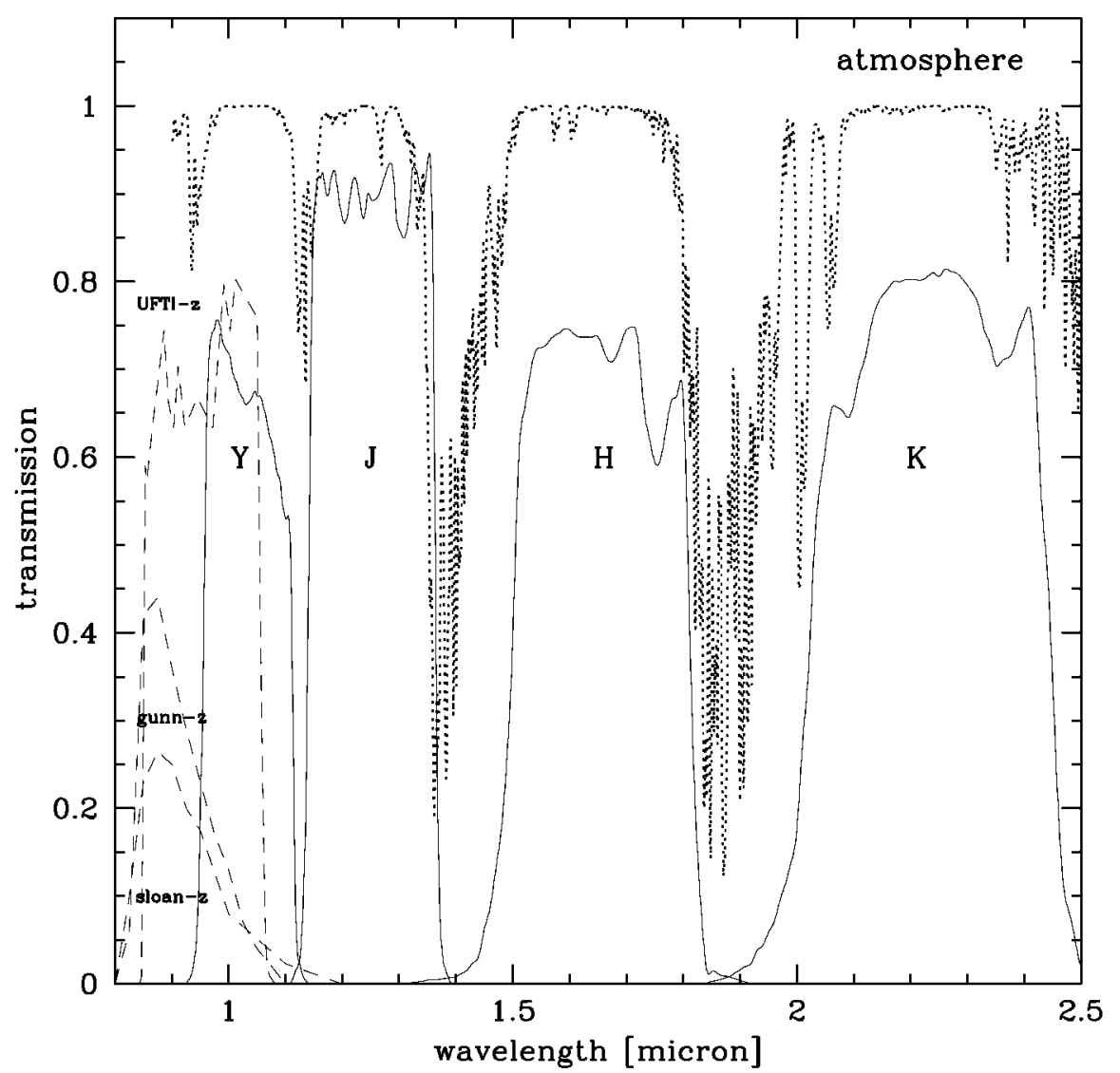

FIG. 1.-Near-infrared $Y, J, H$, and $K$ filter transmission profiles (solid lines) for the Palomar 60 inch cassegrain infrared camera (P60/IRC) filters at $77 \mathrm{~K}$, superposed on atmospheric transmission at Mauna Kea (dotted line). Also shown for comparison to the $Y$-band filter are the optical Gunn $z$ and Sloan $z$ filter profiles convolved with typical CCD response and the UFTI $Z$ filter (cold and already convolved with the Mauna Kea atmospheric transmission as presented in Leggett et al. 2002). The near-infrared $Y$ is longward of previously defined $z$ and $Z$ filters and takes advantage of the relatively clean atmospheric window centered near $1.035 \mu \mathrm{m}$.

sphere above Mauna Kea, produced using the program IRTRANS4 as presented on the UKIRT World Wide Web pages $^{1}$ for an altitude of $4200 \mathrm{~km}$ assuming $1.2 \mathrm{~mm}$ precipitable water vapor and zenith pointing. The $Y$ band is a relatively clean atmospheric window, both in terms of molecular absorption (Fig. 1) and in terms of $\mathrm{O}_{2}$ and $\mathrm{OH}$ night-sky emission lines, which are insignificant in the $Y$ band compared to the $J$ band and $H$ band. Thermal background at the $Y$ band is also insignificant.

The only other filter located in this atmospheric window is, ironically, the F1042M medium/narrowband filter in the Wide Field Planetary Camera 2 (WFPC2) aboard the Hubble Space Telescope (HST), which has $\lambda_{c}=1.015 \mu \mathrm{m}$ and $\delta \lambda_{c}=0.03$ $\mu \mathrm{m}$. Since the original preparation of this manuscript, we have also become aware of the UKIRT Fast Track Imager (UFTI) "Z" filter as described in Leggett et al. (2002). This filter has

\footnotetext{
${ }^{1}$ See http://www.ukirt.jach.hawaii.edu/JACpublic/UKIRT/astronomy/calib/ atmos-index.html.
}

half-power points at 0.851 and $1.056 \mu \mathrm{m}$ (their Table 2), slightly blueward of and broader than our $Y$ filter and inclusive of the strong and broad atmospheric water absorption around $0.93 \mu \mathrm{m}$ (see Fig. 1). The optical "Gunn $z$ " and its successor the "Sloan $z$ " filters (Fukugita et al. 1996) as well as the "Bessel $z$," by contrast, are defined as long-pass filters and hence have their effective profiles determined by a rapidly falling $\mathrm{CCD}$ response curve toward $1 \mu \mathrm{m}$ (Fig. 1). The effective bandpass of these optical $z$ filters is centered near $9250 \AA$ but can vary substantially from instrument to instrument depending on the CCD (e.g., SITe detectors vs. the new red-sensitive Lawrence Berkeley National Laboratories devices as compared in NOAO Newsletter 67, p. 3).

The $Y$ filter introduced here has a well-defined bandpass, as opposed to the long-pass optical $z$ filters, and takes advantage of the relatively clean atmospheric window centered near 1.035 $\mu \mathrm{m}$, in moderate contrast to the UFTI $Z$ filter. $Y$-band photometry therefore should be relatively independent of the specific detector and relatively insensitive to site-to-site differences 


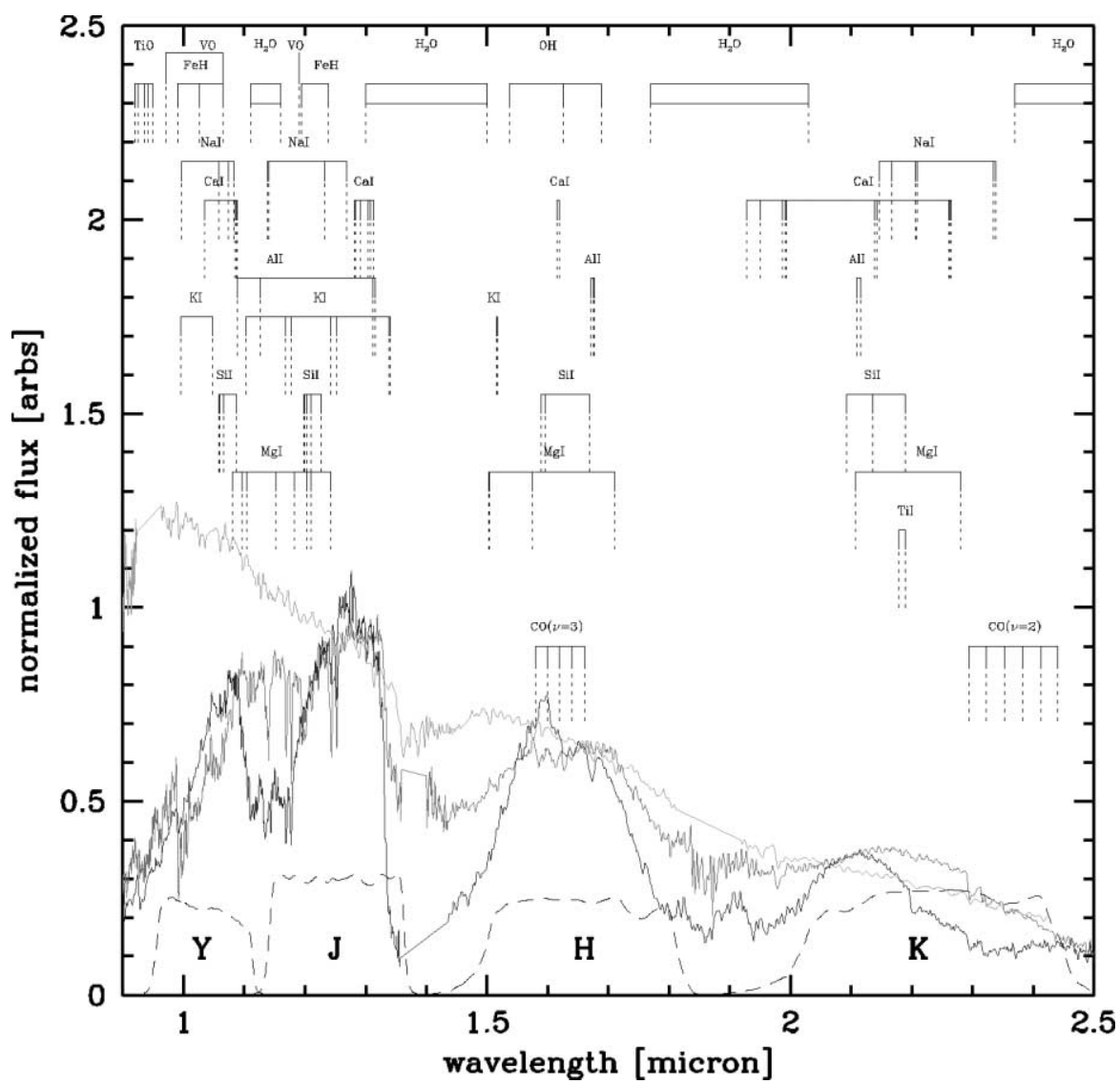

FIG. 2.-Near-infrared $Y, J, H$, and $K$ filter transmission profiles (same as in Fig. 1) superposed on the near-infrared spectra of LHS 386 (M1 V; top), $2 \mathrm{MASS}$ 0746 (L0.5 V; middle), and SDSS 1254 (T2 V; bottom) from Leggett et al. (2000a, 2000b) and Geballe et al. (2002). Top/middle/bottom refers to flux in the $\mathrm{H}_{2} \mathrm{O}$ bands in between the filters; the spectra are arbitrarily normalized at $2.1 \mu \mathrm{m}$. Contributors to spectral absorption features are identified. The near-infrared $Y$ band is located near the peak flux of stars $\sim 3000 \mathrm{~K}$ in temperature ( M4.5-M5 V in spectral type). The peak flux shifts into and remains in the $J$ band at color temperatures down to $500 \mathrm{~K}$ (Burrows et al. 2001), suggesting that $Y-J$ colors may continue to increase relative to Vega's zero color even though $Y-H$ and $Y-K$ (in addition to $J-H$ and $H-K$ ) colors turn blueward for T dwarfs.

in the transparency versus wavelength and the night-to-night variability of water vapor at a given site.

The motivating forces behind the present study are the instrumental/atmospheric advantages of the $1.035 \mu \mathrm{m}$ window just mentioned, combined with the peak brightness of cool and ultracool dwarfs slightly longward of $1 \mu \mathrm{m}$. As shown in Figure 2 , wavelengths $\sim 1 \mu \mathrm{m}$ represent the absolute peak in the spectral energy distributions of low-mass stars with spectral types $\sim$ M5 and a strong local peak for stars and young brown dwarfs with spectral types later than L6 (Burrows et al. 2001). In these very late type dwarfs, the atmospheric opacity redistributes flux far from expectations based on blackbody radiation (Marley et al. 1996). Bolometric corrections become positive in the nearinfrared bands, and $J-H$ and $H-K$ colors become bluer with decreasing effective temperature (Burrows et al. 1997).

Our goals here are to investigate the behavior of near-infrared colors involving the $Y$ band as a function of spectral type and to determine the slope of the interstellar reddening vector. Our specific interest is in assessing the utility of the $Y$ filter in studies of low-mass objects in star-forming regions.

\section{OBSERVATIONS}

Imaging observations were obtained by $\mathrm{L}$. A. $\mathrm{H}$. on the nights of 1999 July 30 and August 1 (UT) using the P60/IRC (Murphy et al. 1995) under photometric sky conditions. The instrument contains a $256 \times 256 \mathrm{HgCdTe}$ NICMOS-3 array with $40 \mu \mathrm{m}$ pixels and produces a final plate scale of 0 "62 pixel $^{-1}$. Observations at $Y$ (called " $X$ " in P60/IRC), $H$, and $K$ were obtained using a nine-point dither pattern centered on target with $10^{\prime \prime}$ separation between adjacent grid points.

Dwarf optical standard stars spanning a range of $B-V$ color from -0.32 (spectral class $\mathrm{O}$ ) to 2.2 (spectral type M6.5) were selected from Landolt (1992) and supplemented at the red end with late-type objects from Kirkpatrick, Henry, \& McCarthy (1991), Tinney (1993), Kirkpatrick, Henry, \& Simons (1995), 
Reid, Hawley, \& Gizis (1995), Hawley, Gizis, \& Reid (1996), Kirkpatrick et al. (1999, 2000), and Burgasser et al. (2002). The latest spectral type observed was L8 V. Infrared standard stars used for calibration of the $H$ and $K$ bands were selected from Persson et al. (1998) and include both unreddened G-type stars and reddened objects of unknown spectral type. A set of eight A0 stars was observed in order to define the instrumental offsets between the $Y$ filter and the $H$ and $K$ filters. The star GSPC S813-D (Lasker et al. 1988) was used to map the atmospheric extinction curve at the $Y$ band. Finally, a moderately reddened field $\left(A_{V}=10-20 \mathrm{mag}\right)$ in the Ophiuchus dark cloud was selected from the large area survey of Barsony et al. (1997) in order to study the interstellar extinction curve at the $Y$ band. On 2001 September 9, some additional data for this program were obtained using the same equipment by A. Burgasser and M. Brown, focusing on $\mathrm{T}$ dwarfs.

Also, observations that predate those above were made of six late $\mathrm{M}$ and $\mathrm{L}$ type dwarfs using the Keck I telescope and NIRC (Matthews \& Soifer 1994) by L. A. H. and J. Carpenter on 1999 February 10 (UT). Details are given in Hillenbrand \& Carpenter (2000). Data of relevance to the present discussion were taken using a five-point dither pattern in the $Y$ (called " $Z$ " in Keck/NIRC) and $K$ filters with appropriate flat-field and dark-current calibration frames also obtained.

\section{DATA REDUCTION}

Our data reduction steps were performed within IRAF and include the standard procedures of applying a linearity correction, masking bad pixels, flat-fielding for correction of sensitivity variations across the array, sky-subtracting, and, finally, mosaicking the dither pattern to produce a final image for photometry. We describe these steps in detail for the P60/IRC observations, although the same basic procedures were followed for the Keck/NIRC data.

Nonlinearity of the detector array was assessed from a series of 2-20 s exposures of the back of the telescope's primary mirror cover taken at the end of the observing run. A central section of the array ( $\frac{1}{4}$ of the total area) that is relatively defectfree was used to measure ADU versus exposure time. The count rate became significantly nonlinear above 36,000 ADU, but to be safe images with a maximum pixel value above 30,000 ADU were discarded.

Below 30,000 counts the response was almost, but not quite, linear. The advertised correction (on the Palomar 60 inch Web page) linearizes data according to

$$
x l=x+x^{2} \times 1.85 \times 10^{-6} \times(1+\delta / \text { EXPTIME }),
$$

where $x l$ represents linear ADU and $x$ raw ADU. For our data, $\delta=3.385$ for an array speed of $1000 \mathrm{~ms}$ and 2.6 for a speed of $743 \mathrm{~ms}$; hence, integration times shorter than $5 \mathrm{~s}$ were avoided during the observations (sometimes by slightly defocusing the telescope when the observing plan called for observations of bright $[<7.5]$ stars) and integrations longer than $10 \mathrm{~s}$ preferred. Different linearization factors were examined, and the correction with the lowest $\chi^{2}$ relative to a linear curve was

$$
x l=x+x^{2} \times 2.1 \times 10^{-6}
$$

without a dependence on integration time for EXPTIME $>5 \mathrm{~s}$. Both the magnitude of this correction and its difference from the standard correction given above are quite small.

Raw bias and flat-field frames were used to verify that the gain and read noise of the detector were similar to the values in Murphy et al. (gain $=8 e^{-}$, read noise $=30 e^{-}$).

Bias frames were acquired as $1.365 \mathrm{~s}$ exposures with the filter wheel in the closed position. Ten frames were averaged after rejecting among the frames the highest and lowest value for each pixel. On each night a sequence of twilight exposures through each filter was used to construct flat-field images. The minimum and maximum values for each pixel were rejected and the individual frames averaged after subtracting the bias image. The sky flat was then normalized to have an overall average value equal to unity. A bad-pixel list was created based on a histogram of the normalized flat-field images by flagging all pixels below 0.65 and above 1.2. This list had considerable overlap with the advertised bad-pixel list, and the two lists were merged into a single bad-pixel mask.

The images of astronomical targets were processed first by constructing sky images as averages of the nine data frames in each dither sequence and subtracting this sky image from each individual data frame, then by determining the relative offsets in fractional pixels between each component of the dither pattern for the point source, and finally by applying the offsets and combining the individual frames with bad-pixel masking into a mosaic. These steps were performed in the NOAO/IRAF environment.

\section{PHOTOMETRY AND CALIBRATION}

We performed aperture photometry with the IRAF/PHOT task. An aperture radius of 12 pixels (15" diameter on the sky) was chosen uniformly for all objects after looking at FWHM values (2-4 pixels) and nearest neighbor distances for the entire data set. The bright A0 stars, which tended to be in crowded fields as a result of proximity to the Galactic plane, set the maximum aperture size. A growth curve of magnitude versus aperture size peaked in the range 11-14 pixels, beyond which the photometric errors increased substantially as the magnitudes decreased for a sky annulus defined from 20-25 pixels. Only for a few (1-2) stars with FWHM just slightly less than 4 pixels (2".5) were the fluxes possibly underestimated using an aperture only $\sim 3$ times their FWHM. The empirical aperture correction for these objects was difficult to find in many cases, but it was examined for several of the largest objects and found to be $\sim 0.007$ mag. Because of the difficulty in determining this 

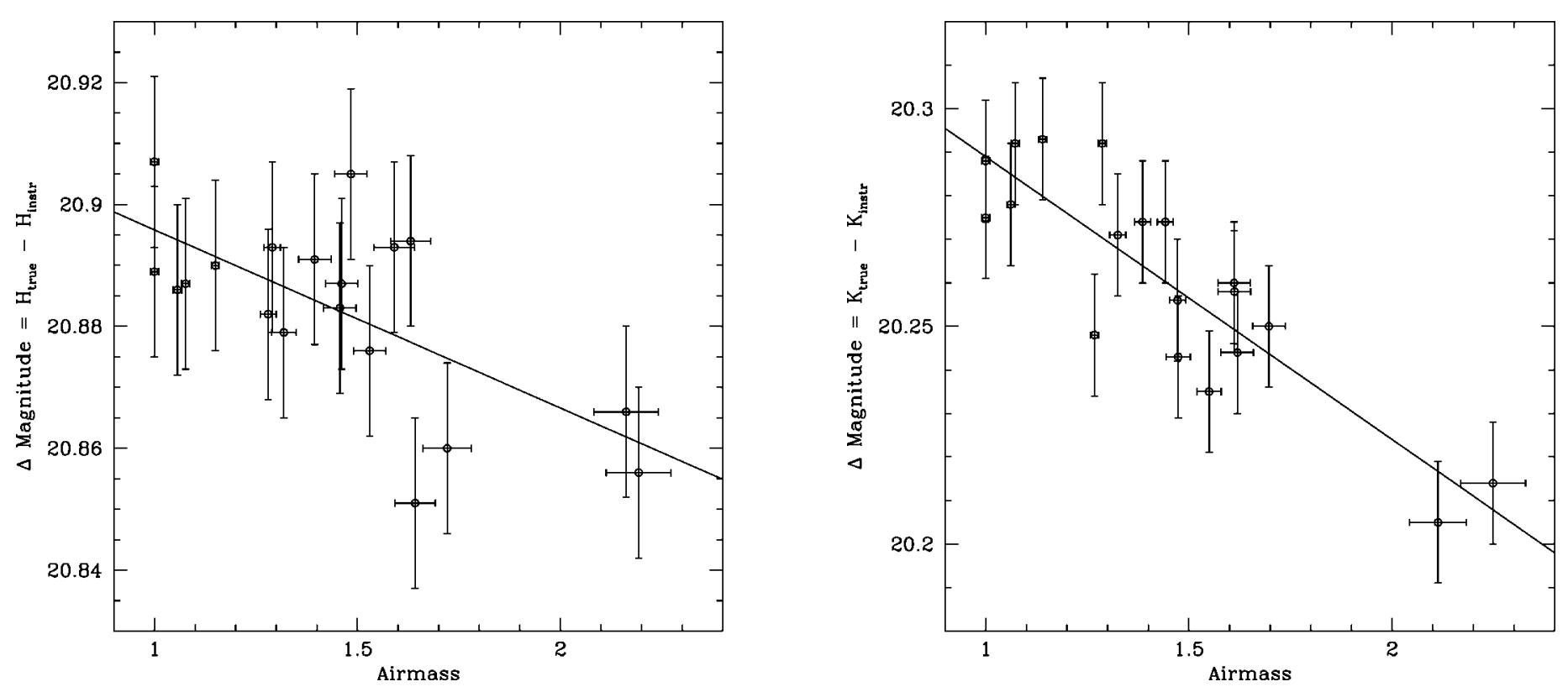

FIG. 3. - Calibration curves for $H$ and $K$ photometry obtained with the Palomar 60 inch telescope and P60/IRC. See text for equations describing the linear fits.

number and its relatively small magnitude, this correction was not applied. Uncertainty in the derived instrumental magnitudes was underestimated by IRAF/PHOT. A better estimate was obtained by examining the photometry of sources in the individual unmosaicked frames where the statistical scatter was observed to be $\sim 0.01 \mathrm{mag}$. We therefore assumed a minimum photometric error of $0.01 \mathrm{mag}$ and characterized our instrumental error as error ${ }_{\text {IRAF/PHOT }}+0.01 \mathrm{mag}$.

The relationship between air mass and (standard - instrumental) magnitude was used to derive the zero points and atmospheric extinction curves for the $K$ and $H$ filters (Fig. 3). Persson et al. (1998) standard stars that were observed over the air-mass range 1.0-2.5, with air mass taken from the middle frame in the nine-position dither pattern, defined these relationships. The star GSPC S813-D was observed at five or six different air masses each night for the explicit purpose of deriving the atmospheric extinction curve for the $Y$ filter without having to know the standard star magnitudes at $Y$ (Fig. 4). Because conditions were photometric, data from both nights were combined in order to obtain a more tightly constrained fit. We now describe the fitting procedure.

While the slopes from each of the two nights were approximately the same at $Y, H$, and $K$, the zero point for the second night was systematically higher by a few hundredths of a magnitude at all wavelengths. In combining data from the two nights, various vertical offsets of night 2 (n2) data to night 1 (n1) data were applied, and the value that minimized the $\chi^{2}$ in the linear fit of the extinction curve was retained. The linear fits assumed errors in air mass given by the half-width of the air mass (AM) range of the observations and errors in the ordinate given by a quadratic sum of our photometry errors (error IRAF/PHOT $+0.01 \mathrm{mag}$ ) plus an arbitrarily assumed 0.01 mag error in the standard star magnitudes.

We thus calibrated the $K$ and $H$ photometry according to

$$
\begin{aligned}
& K_{\text {true }}=K_{\text {instr. }}-(0.065 \pm 0.010) \mathrm{AM}_{K}+\left\{\begin{array}{l}
20.354 \pm 0.015(\mathrm{n} 1) \\
20.389 \pm 0.015(\mathrm{n} 2)
\end{array},\right. \\
& H_{\text {true }}=H_{\text {instr. }}-(0.029 \pm 0.010) \mathrm{AM}_{H}+\left\{\begin{array}{l}
20.925 \pm 0.015(\mathrm{n} 1) \\
20.948 \pm 0.015(\mathrm{n} 2)
\end{array},\right.
\end{aligned}
$$

as shown in Figure 3.

The second term in each equation contains a numerical value and error for the atmospheric extinction, $\kappa_{K, H}$, and the third term values and errors for the zero point, $\mathrm{ZP}_{K, H}$. The extinction coefficients we derive for Palomar, $\kappa_{K}=0.065$ and $\kappa_{H}=0.029$, agree well with those from the 2MASS survey ${ }^{2}$ at Mount Hopkins a few hundred miles away and a few thousand feet higher, $\kappa_{K s}=0.061$ and $\kappa_{H}=0.031$. Note that our quoted zero points are for $\mathrm{AM}=0$, i.e., outside the atmosphere, assuming linear extrapolation of the fit derived from AM $>1$; see Figure 3 of Tokunaga, Simons, \& Vacca (2002) for the (in)appropriateness of this extrapolation in various near-infrared bandpasses.

To calibrate the $Y$ filter, we assume by definition that unreddened A0 stars have zero color. The A0 stars observed for this program are lightly reddened as a result of the requirement that they be faint enough to observe with P60/IRC (implying $K>7.5 \mathrm{mag}$ ), which is essentially a minimum distance requirement given the absolute magnitude of an A0 star, and hence a minimum reddening requirement given the properties

\footnotetext{
${ }^{2}$ See http://www.ipac.caltech.edu/2mass/releases/first/doc/sec4_8.html.
} 
TABLE 2

Photometry of Optical/Infrared Standard Stars and Additional Red Objects

\begin{tabular}{|c|c|c|c|c|c|c|c|c|c|c|c|c|c|c|}
\hline Source Name & Spectral Type & $B-V$ & $V-I$ & $I-K$ & $Y$ & $Y$ Error & $Y-H$ & $Y-H$ Error & $Y-K$ & $Y-K$ Error & $J_{2 \text { MASS }}$ & $H_{2 \mathrm{MASS}}$ & $K_{\text {2MASS }}$ & Comment \\
\hline PG 2213-006 & $\mathrm{sdO}$ & -0.217 & -0.203 & -0.378 & 14.559 & 0.030 & -0.132 & 0.048 & -0.167 & 0.108 & 14.70 & 14.78 & 14.60 & Landolt \\
\hline HD $218902 \ldots$ & A0 V & -0.01 & $\ldots$ & $\ldots$ & 10.244 & 0.036 & -0.011 & 0.045 & -0.020 & 0.046 & 10.23 & 10.22 & 10.20 & Zero point \\
\hline HD 222644 & $\mathrm{~A} 0 / \mathrm{A} 1 \mathrm{~V}$ & $0.1:$ & & & 10.571 & 0.032 & 0.010 & 0.040 & -0.004 & 0.040 & 10.56 & 10.54 & 10.54 & Zero point \\
\hline SA $112-805$ & $\mathrm{~A} 0 / \mathrm{A} 1$ & 0.152 & 0.138 & 0.205 & 11.836 & 0.030 & 0.051 & 0.038 & 0.065 & 0.041 & & & & Landolt/zero point \\
\hline HD $191398 \ldots$ & A0 V & 0.09 & $\ldots$ & $\ldots$ & 8.739 & 0.026 & 0.084 & 0.033 & 0.094 & 0.033 & 8.67 & 8.60 & 8.61 & Zero point \\
\hline HD 228700 & $\mathrm{~A} 0 \mathrm{~V}$ & 0.11 & $\ldots$ & $\ldots$ & 8.905 & 0.026 & 0.119 & 0.033 & 0.126 & 0.034 & 9.39 & 9.33 & 9.31 & Zero point \\
\hline HD 161481 & $\mathrm{~A} 0$ & 0.24 & $\ldots$ & $\ldots$ & 8.594 & 0.029 & 0.157 & 0.036 & 0.156 & 0.037 & $\ldots$ & & & Zero point \\
\hline HD 161168 & A0 & $0.3:$ & $\ldots$ & $\ldots$ & 8.856 & 0.038 & 0.109 & 0.047 & 0.163 & 0.048 & 8.81 & 8.77 & 8.70 & Zero point \\
\hline SA $109-71$ & $\mathrm{~A} 0$ & 0.323 & 0.41 & 0.496 & 10.823 & 0.035 & 0.170 & 0.044 & 0.206 & 0.045 & $\ldots$ & $\ldots$ & $\ldots$ & Landolt/zero point \\
\hline HD $167163 \ldots$ & A $0 \mathrm{~V}$ & 0.35 & $\ldots$ & $\ldots$ & 8.705 & 0.029 & 0.179 & 0.037 & 0.222 & 0.037 & $\ldots$ & $\ldots$ & $\ldots$ & Zero point \\
\hline SA $115-420 \ldots \ldots \ldots \ldots$ & F5 & 0.468 & 0.58 & 0.657 & 10.362 & 0.027 & 0.392 & 0.035 & 0.399 & 0.036 & $\ldots$ & $\ldots$ & $\ldots$ & Landolt \\
\hline GSPC P576-F = SJ 9183 & $\mathrm{G}$ & $\ldots$ & $\ldots$ & $\ldots$ & 12.296 & 0.030 & $\ldots$ & & 0.419 & 0.042 & $\ldots$ & $\ldots$ & $\ldots$ & Persson \\
\hline GSPC P550-C = SJ 9143 & $\mathrm{G}$ & $\ldots$ & $\ldots$ & $\ldots$ & 12.610 & 0.05 & 0.493 & 0.05 & 0.542 & 0.05 & $\ldots$ & $\ldots$ & $\ldots$ & Persson \\
\hline SA $113-492 \ldots \ldots \ldots \ldots$ & G0: & 0.553 & 0.684 & 0.785 & 11.279 & 0.028 & 0.545 & 0.036 & 0.546 & 0.038 & $\ldots$ & $\ldots$ & $\ldots$ & Landolt \\
\hline GSPC P290-D = SJ 9188 & $\mathrm{G}$ & $\ldots$ & $\ldots$ & $\ldots$ & 11.875 & 0.026 & 0.524 & 0.034 & 0.581 & 0.036 & & & & Persson \\
\hline GSPC P330-E = SJ 9166 & $\mathrm{G}$ & $\ldots$ & $\ldots$ & $\ldots$ & 12.041 & 0.018 & 0.565 & 0.022 & 0.582 & 0.023 & 11.79 & 11.46 & 11.43 & Persson \\
\hline FS $35, \ldots \ldots \ldots \ldots$ & K0 & 0.8: & $\ldots$ & $\ldots$ & 12.359 & 0.030 & $0.52^{\mathrm{b}}$ & 0.030 & 0.582 & 0.030 & $12.20^{\mathrm{c}}$ & $11.83^{\mathrm{c}}$ & $11.75^{\mathrm{c}}$ & Hawarden \\
\hline GSPC S813-D = SJ 9182 & $\mathrm{G}$ & $\ldots$ & $\ldots$ & $\ldots$ & 11.697 & 0.009 & 0.552 & 0.010 & 0.586 & 0.010 & 11.47 & 11.12 & 11.13 & Persson \\
\hline GSPC P545-C $=$ SJ $9134^{\mathrm{a}}$ & G & $\ldots$ & $\ldots$ & $\ldots$ & 12.155 & 0.05 & 0.531 & 0.05 & 0.598 & 0.05 & 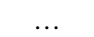 & & & Persson \\
\hline GSPC P182-E = SJ 9177. & $\mathrm{G}$ & $\ldots$ & $\ldots$ & $\ldots$ & 12.328 & 0.016 & 0.558 & 0.020 & 0.603 & 0.021 & 12.09 & 11.76 & 11.71 & Persson \\
\hline SA $113-493 \ldots \ldots \ldots \ldots$ & G5: & 0.786 & 0.824 & 0.97 & 10.666 & 0.028 & 0.608 & 0.036 & 0.664 & 0.037 & $\ldots$ & $\ldots$ & $\ldots$ & Landolt \\
\hline SA $109-949, \ldots \ldots \ldots \ldots$ & G8: & 0.806 & 1.02 & 1.130 & 11.384 & 0.029 & 0.595 & 0.037 & 0.668 & 0.038 & $\ldots$ & . & & Landolt \\
\hline GSPC S867-V = SJ 9155 & G5 & 0.68 & $\ldots$ & $\ldots$ & 12.363 & 0.05 & 0.719 & 0.05 & 0.729 & 0.05 & $11.98^{\mathrm{c}}$ & $11.64^{\mathrm{c}}$ & $11.59^{\mathrm{c}}$ & Persson/Hawarden \\
\hline 2MASSI J2254188+312349 & $\mathrm{T} 5 \mathrm{~V}$ & $\ldots$ & $\ldots$ & $\ldots$ & 16.037 & 0.051 & $1.00^{\mathrm{b}}$ & 0.051 & 0.823 & 0.092 & 15.28 & 15.04 & 14.83 & Late type \\
\hline Gl $229 b^{\mathrm{d}} \ldots \ldots \ldots \ldots \ldots \ldots \ldots$ & $\mathrm{T} 6.5 \mathrm{~V}$ & $\ldots$ & $\ldots$ & $>5.6$ & 15.3 & $\ldots$ & 1.0 & & 0.9 & & $\ldots$ & $\ldots$ & $\ldots$ & Late type \\
\hline SA $113-495 \quad \ldots \ldots \ldots$ & G: & 0.947 & 1.01 & 1.306 & 11.055 & 0.028 & 0.849 & 0.036 & 0.906 & 0.037 & $\ldots$ & $\ldots$ & $\ldots$ & Landolt \\
\hline SA $110-477$ & $\ldots$ & 1.345 & 1.707 & 1.685 & 11.572 & 0.036 & 0.823 & 0.045 & 0.947 & 0.046 & $\ldots$ & $\ldots$ & $\ldots$ & Landolt \\
\hline SA $109-956$ & $\ldots$ & 1.283 & 1.525 & 1.796 & 12.516 & 0.029 & 1.038 & 0.038 & 1.160 & 0.041 & $\ldots$ & $\ldots$ & & Landolt \\
\hline Gl 754.1B ... & M2.5 V & 1.469 & 2.87 & 1.475 & 8.547 & 0.037 & 0.964 & 0.045 & 1.193 & 0.045 & 9.54: & 9.63 & 9.01: & Late type \\
\hline SA $109-954 \ldots \ldots \ldots$ & & 1.296 & 1.496 & 1.929 & 10.254 & 0.028 & 1.064 & 0.036 & 1.204 & 0.037 & & & & Landolt \\
\hline LHS $3409=$ GJ 4076. & M3.5 V-sdM4.5 & 1.85 & 2.66 & 2.223 & 11.486 & 0.027 & 0.964 & 0.034 & 1.211 & 0.035 & $10.98^{\mathrm{c}}$ & $10.49^{\mathrm{c}}$ & $10.26^{\mathrm{c}}$ & Late type \\
\hline GJ $1220=$ LHS $3297 \ldots \ldots \ldots \ldots \ldots \ldots \ldots$ & M4 V & 1.73 & 2.96 & 2.489 & 10.128 & 0.032 & 1.083 & 0.040 & 1.339 & 0.040 & $\ldots$ & $\ldots$ & $\ldots$ & Late type \\
\hline GJ $1215=$ LHS $3277 \ldots \ldots \ldots \ldots \ldots \ldots \ldots$ & M5.5 V & 1.96 & 3.32 & 2.875 & 10.377 & 0.032 & 1.105 & 0.040 & 1.404 & 0.040 & $\ldots$ & $\ldots$ & $\ldots$ & Late type \\
\hline 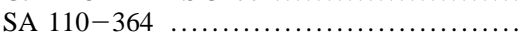 & $\ldots$ & 1.133 & 1.281 & 1.996 & 11.775 & 0.035 & 1.120 & 0.043 & 1.409 & 0.044 & $\ldots$ & $\ldots$ & $\ldots$ & Landolt \\
\hline
\end{tabular}




\begin{tabular}{|c|c|c|c|c|c|c|c|c|c|c|c|c|c|c|}
\hline LHS $523=$ GJ $4281 \ldots \ldots \ldots \ldots$ & M6.5 V & 2.03 & 4.26 & 3.133 & 11.354 & 0.030 & 1.134 & 0.039 & 1.449 & 0.040 & $10.79^{c}$ & $10.22^{\mathrm{c}}$ & $9.91^{\mathrm{c}}$ & Late type \\
\hline BRI $2202-1119=$ LP $759-25$. & M6.5 V & & $\ldots$ & 2.936 & 12.226 & 0.032 & 1.158 & 0.040 & 1.464 & 0.040 & 11.68 & 11.06 & 10.73 & Late type \\
\hline TVLM $868-53850 \quad \ldots \ldots \ldots \ldots \ldots$ & M6: & & & & 12.195 & 0.031 & 1.197 & 0.039 & 1.504 & 0.040 & 11.53 & 10.94 & 10.65 & Persson \\
\hline VB $8 \ldots \ldots \ldots \ldots \ldots$ & M7 V & 2.2 & 4.56 & 3.405 & 10.455 & 0.033 & 1.232 & 0.042 & 1.591 & 0.042 & & & & Late type \\
\hline LHS $2632=$ LP $321-222^{a} \ldots \ldots \ldots \ldots \ldots \ldots$ & M7.5 V & & & & 13.001 & 0.05 & $1.41^{\mathrm{b}}$ & 0.05 & $1.77^{\mathrm{b}}$ & 0.05 & 12.26 & 11.59 & 11.23 & Late type \\
\hline LHS $3003=$ GJ $3877^{\mathrm{a}} \ldots \ldots$ & M7 V & 1.34 & 4.52 & 3.196 & 10.735 & 0.05 & $1.41^{\mathrm{b}}$ & 0.05 & 1.815 & 0.05 & 9.96 & 9.32 & 8.92 & Late type \\
\hline 2MASS J1524248+292535 & & $\ldots$ & $\ldots$ & $\ldots$ & 12.069 & 0.05 & & & 1.847 & 0.05 & & & & Late type \\
\hline SDSSp $175032.96+175903.9 \ldots \ldots \ldots \ldots \ldots \ldots$ & T3.5 & $\ldots$ & $\ldots$ & $\ldots$ & 17.427 & 0.132 & $1.49^{\mathrm{b}}$ & 0.132 & $1.96^{\mathrm{b}}$ & 0.132 & 16.32 & 15.94 & 15.47 & Late type \\
\hline SDSSp $015141.69+124429.6 \ldots \ldots \ldots \ldots \ldots$ & $\mathrm{T} 1$ & $\ldots$ & $\ldots$ & $\ldots$ & 17.173 & 0.111 & $1.63^{\mathrm{b}}$ & 0.111 & 1.965 & 0.138 & 16.25 & 15.54 & 15.18 & Late type \\
\hline TVLM $868-110639 \ldots \ldots \ldots \ldots$ & M9 V & $\ldots$ & $\ldots$ & 4.422 & 13.470 & 0.033 & 1.584 & 0.042 & 2.064 & 0.043 & & & & Persson \\
\hline LHS $2924=$ GJ $3849^{\mathrm{a}} \ldots \ldots \ldots \ldots \ldots \ldots \ldots \ldots$ & M9 V & 1.62: & 4.37 & 4.446 & 12.940 & 0.05 & $1.77^{\mathrm{b}}$ & 0.05 & 2.176 & 0.05 & $11.92^{\mathrm{c}}$ & $11.17^{\mathrm{c}}$ & $10.75^{\mathrm{c}}$ & Late type \\
\hline 2MASSW J1439284+192915 a & L1 V & $\ldots$ & 4.92 & 4.53 & 13.778 & 0.05 & $1.73^{\mathrm{b}}$ & 0.05 & 2.187 & 0.05 & 12.76 & 12.05 & 11.58 & Late type \\
\hline 2MASSW J0030438+313932 $\ldots$ & L2 V & $\ldots$ & $\ldots$ & & 16.448 & 0.033 & 1.864 & 0.047 & 2.418 & 0.078 & 15.49 & 14.58 & 13.99 & Late type \\
\hline DENIS-P J0205.4-1159 _................... & L7 V & $\ldots$ & $\ldots$ & 5.31 & 15.425 & 0.037 & $1.84^{\mathrm{b}}$ & 0.037 & 2.437 & 0.042 & 14.58 & 13.59 & 12.98 & Late type \\
\hline 2MASSW J2208136+292121 & L2 V & $\ldots$ & $\ldots$ & 5.66 & 16.894 & 0.068 & $2.06^{\mathrm{b}}$ & 0.068 & 2.826 & 0.076 & 15.82 & 14.83 & 14.09 & Late type \\
\hline 2MASSW J1728114+394859 ….......... & L7 V & $\ldots$ & $\ldots$ & $\ldots$ & 16.821 & 0.064 & $2.04^{\mathrm{b}}$ & 0.064 & $2.92^{\mathrm{b}}$ & 0.064 & 15.96 & 14.78 & 13.90 & Late type \\
\hline 2MASSI J1029216+162652 $\ldots \ldots \ldots \ldots \ldots \ldots$ & L2.5 & $\ldots$ & $\ldots$ & & 15.565 & 0.05 & $2.22^{\mathrm{b}}$ & 0.05 & 2.952 & 0.05 & 14.31 & 13.35 & 12.61 & Late type \\
\hline 2MASSW J1632291+190441 $\ldots \ldots \ldots \ldots \ldots$ & L8 V & $\ldots$ & $\ldots$ & $>5.16$ & 16.984 & 0.043 & 2.260 & 0.059 & 2.993 & 0.081 & 15.86 & 14.59 & 13.98 & Late type \\
\hline 2MASSI J0103320+193536 _............... & L6 V & $\ldots$ & $\ldots$ & $\ldots$ & 17.312 & 0.123 & $2.43^{\mathrm{b}}$ & 0.123 & 3.169 & 0.127 & 16.26 & 14.88 & 14.15 & Late type \\
\hline 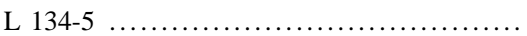 & $\ldots$ & $\ldots$ & $\ldots$ & $\ldots$ & 12.976 & 0.033 & 3.296 & 0.041 & 4.012 & 0.041 & & $\ldots$ & & Persson/extincted \\
\hline "Oph2" = 2MASS J162705-242010 $\ldots \ldots \ldots$ & $\ldots$ & $\ldots$ & $\ldots$ & $\ldots$ & 17.530 & 0.094 & 3.677 & 0.105 & 4.612 & 0.109 & 12.70 & 10.50 & 9.36 & Extincted \\
\hline "Oph5" = 2MASS J162705-242036 . & $\ldots$ & $\ldots$ & $\ldots$ & $\ldots$ & 14.813 & 0.038 & 3.724 & 0.047 & 4.668 & 0.047 & & 14.47 & 12.97 & Extincted \\
\hline 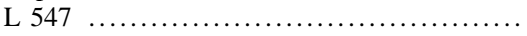 & $\ldots$ & $\ldots$ & $\ldots$ & $\ldots$ & 13.750 & 0.030 & 3.833 & 0.037 & 4.741 & 0.037 & 10.83 & 10.39 & 10.25 & Persson/extincted \\
\hline "Oph9" = 2MASS J162703-241832 …... & $\ldots$ & $\ldots$ & $\ldots$ & $\ldots$ & 18.408 & 0.118 & 3.647 & 0.137 & 4.907 & 0.136 & $\ldots$ & $\ldots$ & $\ldots$ & Extincted \\
\hline "Oph8" = 2MASS J162707-242009 ....... & $\ldots$ & & & $\ldots$ & 14.582 & 0.038 & 4.117 & 0.046 & 5.186 & 0.046 & $\ldots$ & $\cdots$ & $\cdots$ & Extincted \\
\hline "Oph6" = 2MASS J162706-241923 $\ldots \ldots \ldots$ & $\ldots$ & $\ldots$ & $\ldots$ & $\ldots$ & 19.248 & 0.309 & 4.202 & 0.320 & 5.363 & 0.321 & $\ldots$ & 14.86 & 13.83 & Extincted \\
\hline "Oph11" = 2MASS J162658-241837 ...... & $\ldots$ & $\ldots$ & $\ldots$ & $\ldots$ & 18.758 & 0.167 & 4.120 & 0.179 & 5.363 & 0.179 & $\ldots$ & 14.51 & 13.34 & Extincted \\
\hline "Oph3" = 2MASS J162702-242040 $\ldots \ldots \ldots$ & $\ldots$ & $\ldots$ & $\ldots$ & $\ldots$ & 17.519 & 0.111 & 4.518 & 0.117 & 5.883 & 0.117 & $\ldots$ & $\ldots$ & $\ldots$ & Extincted \\
\hline
\end{tabular}

Note. - Table is sorted by $Y-K$ color. Spectral types and luminosity classes are given where available and taken, along with $B-V$ and $V-I$ photometry from the literature. $I-K$ colors are calculated by combining literature $I$ with the present $K$ measurements. $Y, Y-H$, and $Y-K$ photometry is newly measured here. Errors include terms for zero point, atmospheric extinction, and photon statistics added in quadrature. $J_{\text {2MASS }}, H_{2 \mathrm{MASS}}$, and $K_{\text {2MAss }}$ values come from the 2MASS Second Incremental Release and have errors typically $0.03-0.04$ mag. Comment indicates the type of object, either a photometric standard star from Landolt 1992, Persson et al. 1998, or Hawarden et al. 2001; a later type usually spectroscopic standard star; or an extincted/reddened object.

$Y H K$ data is from Keck/NIRC instead of P60/IRC.

Star's quoted $Y-H$ or $Y-K$ color is from measured $Y$ magnitude and 2MASS or other $H, K / K_{s}$ photometry.

c Star's JHK photometry is from Geballe et al 2002, Hawarden et al. 2001, Leggett, Allard, \& Hauschildt 1998, or Leggett 1992 (largely in MKO/UKIRT system) instead of from 2MASS.

${ }^{\mathrm{d}}$ Gl $229 \mathrm{~b}$ photometry is from Nakajima et al. 1995 and Matthews et al. 1996. 


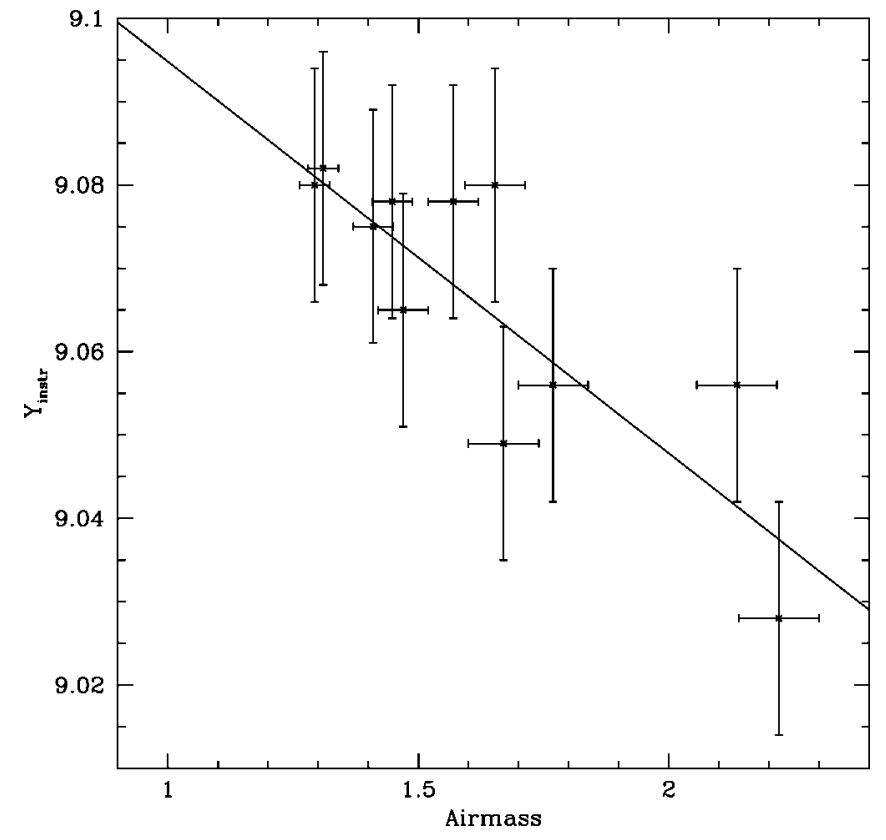

FIG. 4.-Instrumental magnitude in the $Y$ filter vs. air mass for GSPC S813D. Data for both nights on which P60/IRC data were obtained were combined, with the second night's values shifted by 0.035 mag to produce the lowest error in the linear fit.

of the local interstellar medium. We therefore include an interstellar extinction term in our calibration equations. We used both the $K$-band and the $H$-band data independently to derive the zero point at $Y$, from

$$
-K_{\text {instr. }}+Y_{\text {instr. }}=\mathrm{ZP}_{K}-\mathrm{ZP}_{Y}-\left(\kappa_{K}-\kappa_{Y}\right) \mathrm{AM}-\left(\frac{A_{K}}{A_{V}}-\frac{A_{Y}}{A_{V}}\right) A_{V},
$$

and a similar equation extrapolating to the $Y$ band from the $H$ band instead of the $K$ band. The difference between the two approaches is less than $0.015 \mathrm{mag}$ and within the $\sim 1 \sigma$ A0 star photometry errors. Above, $Y_{\text {instr. }}$ is the instrumental magnitude, $\mathrm{ZP}_{Y}$ is the zero point, $\kappa_{Y}$ is the slope of the atmospheric extinction curve, and $A_{Y}$ is the interstellar extinction. To find $\kappa_{Y}$ we combined the data for GSPC S813-D from both nights, shifting the night 2 data by $0.035 \mathrm{mag}$ to obtain the best fit to a straight line, giving $\kappa_{Y}=0.047$. Figure 4 shows this slope determination. For the interstellar extinction term we use literature or Tycho $B-V$ photometry and assume $A_{V}=3.1 E(B-V), A_{Y}=0.38 A_{V}$ (anticipating our derivation of this result below in $\S 5.2), A_{H}=0.155 A_{V}$, and $A_{K}=$ $0.090 A_{V}$ (Cohen et al. 1981). The two A0 stars with less well determined $B-V$ photometry (see Table 2) were left out of the final analysis since they introduced larger scatter and a systematic shift in the results between the $H$ and $K$ derivations.

With $\kappa_{Y}$ and $A_{Y}$ in hand, the above equation and an analogous one for $H$ are solved for $\mathrm{ZP}_{Y}$; errors are calculated by summing in quadrature the errors from each term of the equations. From this solution for $\mathrm{ZP}_{Y}$ we then correct the 10-12 data points for GSPC S813-D and combine these measurements with the A0 points to derive the final $Y$-band calibration equation:

$$
Y_{\text {true }}=Y_{\text {instr. }}-(0.047 \pm 0.018) \mathrm{AM}_{Y}+\left\{\begin{array}{lll}
20.835 \pm 0.030 & (\mathrm{n} 1) \\
20.875 \pm 0.030 & (\mathrm{n} 2)
\end{array} .\right.
$$

The zero points at $Y$ derived from $K$ and from $H$ agree to well within the estimated errors, differing by only $0.015 \mathrm{mag}$, and the quoted numbers are the averages of the two approaches. The $\chi^{2}$ values for the fits are 0.8 and 0.7 for the extrapolation from $K$ and $H$, respectively, a little less than unity, which indicates a good assessment of errors. The difference in zero point between the two nights is $0.040 \mathrm{mag}$, whereas we had shifted the data for S813-D between the two nights by 0.035 to derive the slope of atmospheric extinction, all consistent, and suggestive that only a single iteration of the procedure is needed.

The final $Y, Y-H$, and $Y-K$ photometry for all objects is presented in Table 2 along with errors and ancillary information. These measurements are in the natural system of the camera, which should be that defined by the Persson et al. (1998) standards set up in part using this same camera. Magnitude uncertainties are given by

$$
\sigma_{\text {magnitude }}=\sqrt{\sigma_{\mathrm{ZP}}^{2}+\mathrm{AM}^{2} \sigma_{\kappa}^{2}+\sigma_{\text {instr. }}^{2}},
$$

which contains $1 \sigma$ error terms for the zero point, atmospheric extinction assuming no uncertainty in the recorded air mass, and instrumental errors $\sigma_{\text {instr. }}=\left(\sigma_{\text {IRAF/PHOT }}+0.01 \mathrm{mag}\right)$. Color errors are assumed to be the root sum square of the relevant magnitude errors, which means that we are ignoring the effects of any correlated errors in our photometry. Multiple measurements of the same star were averaged, mostly occurring in the case of GSPC standards. Also given in Table 2 are optical photometry and spectral types from the literature and 2MASS photometry from the Second Incremental Release for comparison with our $H$ and $K$ measurements.

\section{ANALYSIS}

\subsection{Colors}

Color-color diagrams were made for different color combinations using the $H$ and $K$ results presented in this paper and $B, V$, and $I$ photometry obtained from the literature where available. These plots were superposed on standard color-color lines (e.g., Bessell \& Brett [1988] transformed to the CIT photometric system), and good agreement was seen between our $H$ and $K$ photometry and expected $I-K$ and $H-K$ colors. Moving now to the $Y$ filter, Figure 5 shows the infrared $Y-K$ color compared to optical $B-V$ and $V-I$ colors. These colors redden together with increasing spectral type to late $\mathrm{M}$ or early $\mathrm{L}$, at which point the optical colors become saturated and/or turn bluer while the infrared color continues to grow redder. Note 

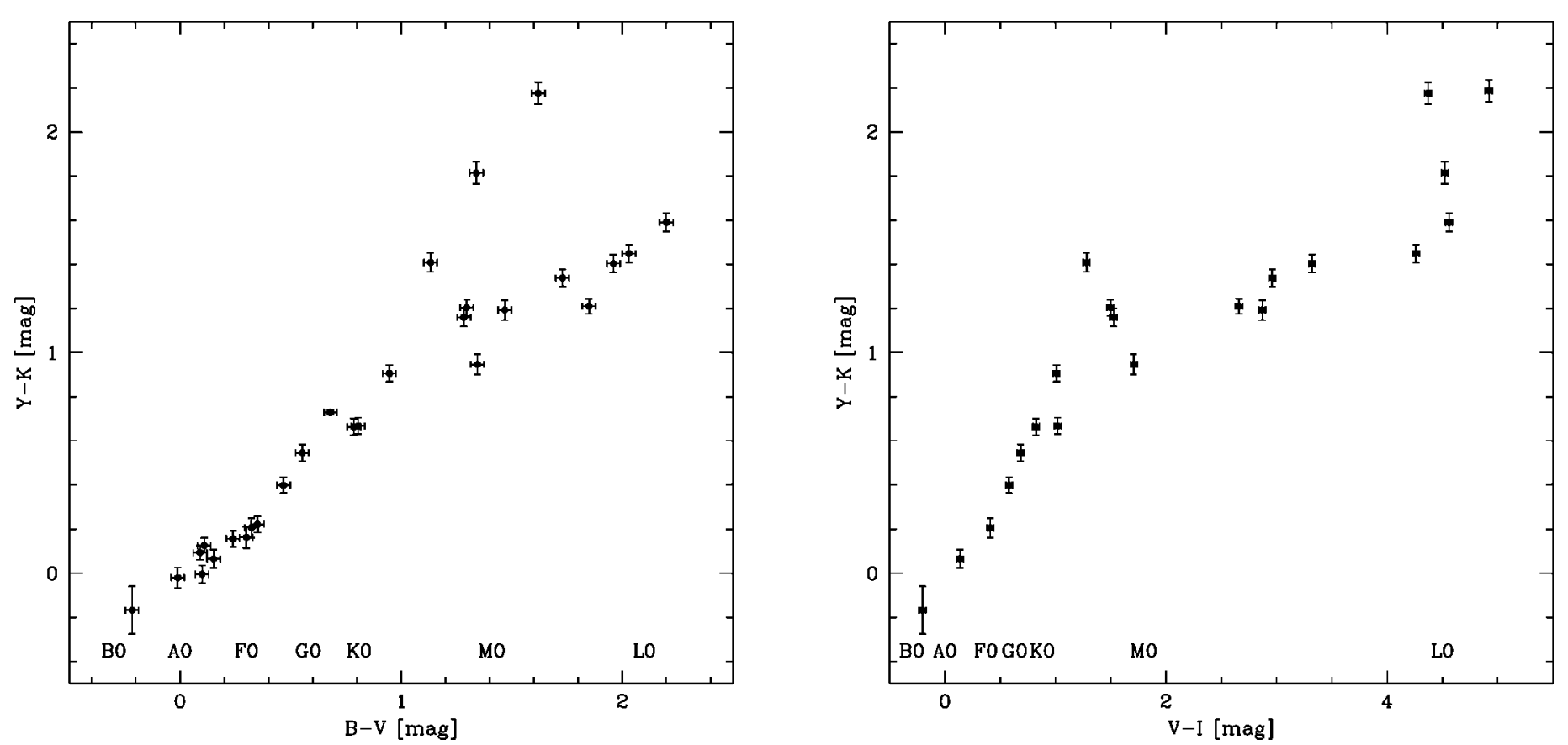

FIG. 5.-New near-infrared $Y-K$ colors compared to optical $B-V(l e f t)$ and $V-I$ (right) colors from the literature. Locations of spectral type labels correspond to optical colors. The reddest stars in $Y-K$ with published optical photometry appearing in these figures are 2MASSW 1439284+192915 (L1 in the $V-I$ plot only, with $Y-K=2.19$ ), LHS 2924 (M9, with $Y-K=2.18$ ), LHS 3003 (M7 or perhaps later, with $Y-K=1.82$ ), and VB 8 (M7, with $Y-K=1.59$ ). $B-V$ colors appear to saturate and turn blueward around spectral type M7, while $V-I$ colors saturate around spectral type M8 but do not turn systematically blueward at late types.

that optical colors are not available for all of the objects in Table 2, particularly the later type, redder stars and brown dwarfs.

Plots of $Y-H$ versus $H-K, Y-K$ versus $H-K$, and $Y-H$ versus $Y-K$ were then investigated, as seen in Figures 6 and 7. The range of spectral types extends from sdO through T5 V, with photometry for the T6.5 V brown dwarf G1 229b (Matthews et al. 1996) shown for comparison. Gaps in the data around $Y-K=0.3$ and $0.8 \mathrm{mag}$ are due to the lack of $\mathrm{F}$ and $\mathrm{K}$ stars in our sample. These plots reveal a relatively tight relationship between the $Y H K$ colors as they grow redder together through spectral type $\sim \mathrm{L} 6 \mathrm{~V}$. Beyond L6 V, the colors turn blueward, and from $\mathrm{L} 7 \mathrm{~V}$ to at least $\mathrm{T} 6.5 \mathrm{~V}$ the colors are nearly degenerate with those of warmer L and M stars.

There are six objects in common between this study and that of Leggett et al. (2002): LHS 3003 (M7 V), LHS 2924 (M9 V), 2MASS J1439284+192915 (L1 V), DENIS-P J0205.4-1159 (L7 V), 2MASS 1632291+190441 (L8 V), and SDSSp 015141.69+124429.6 (T1). Our $Y$-band magnitudes are 0.3-0.6 mag brighter than their UFTI $Z$ magnitudes, and our colors are accordingly bluer for these objects, consistent with the nature of the filter center and width differences described in our introduction.

\subsection{Extinction}

In order to study the effects of interstellar extinction on colors involving the $Y$-band filter, a moderately reddened field toward the edge of the Ophiuchus dark cloud was observed. Eleven stars were identified in all three filter images, but only seven were bright enough in $Y$ to produce acceptable photometry. L134 and L547 from Persson et al. (1998) were also included in this analysis. The locations of these nine stars in $Y H K$ colorcolor diagrams did not enable us to deduce the magnitude of the reddening vector, as they lay basically along an extrapolation of the color-color line defined by the dwarf stellar/ substellar sequence (see Figs. 6 and 7).

In order to quantify how objects redden in the $Y$ band relative to the $V$ band, we retrieved $J, H$, and $K_{s}$ photometry for the seven Ophiuchus and the two Persson "red objects" from the 2MASS Second Incremental Release. We then dereddened these nine stars using the 2MASS $J-H$ and $H-K_{s}$ colors and the Cohen et al. (1981) interstellar extinction curve until they intersected the dwarf color sequence in a $J-H$ versus $H-K_{s}$ diagram over the color range corresponding to spectral types K7-M6. Fortunately, these stars all appear to be background stars and not young stellar objects associated with the Ophiuchus (and other, in the case of the Persson et al. "red stars") clouds, although we cannot prove this. If they were indeed located within these clouds, they could have circumstellar dust/gas and hence colors that reflect substantial near-infrared excess in addition to the effects of reddening, invalidating our approach. The estimated $H-K$ and $J-H$ color excess due to interstellar reddening was converted to $A_{V}$. 


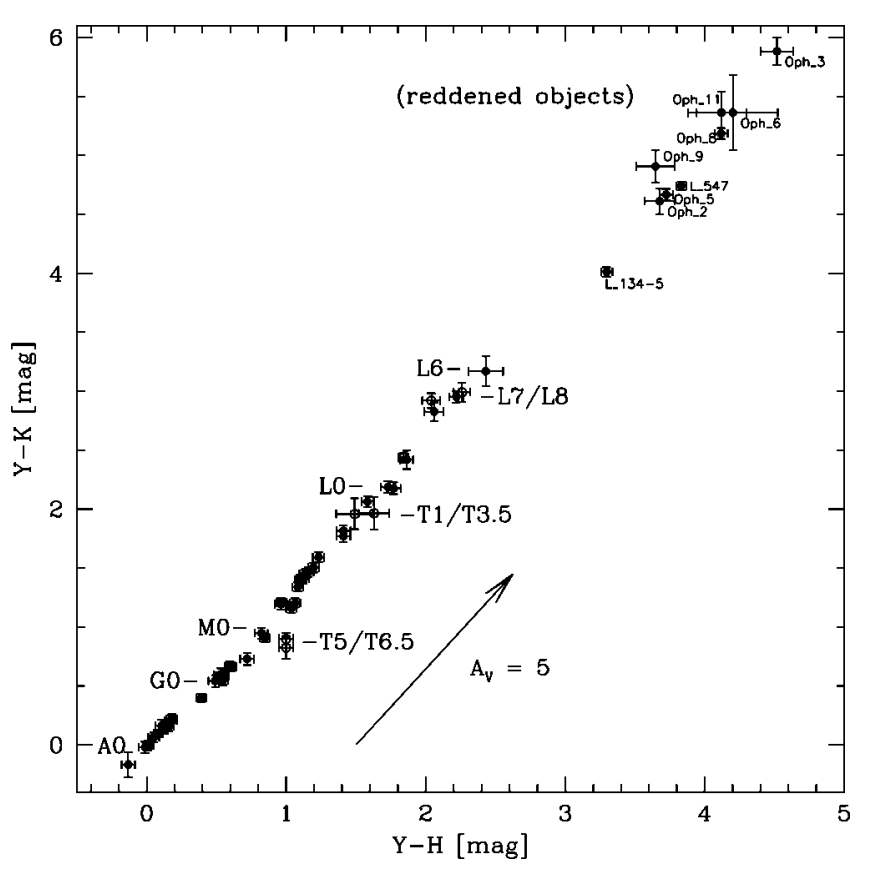

Fig. 6.-Newly derived $Y-K$ vs. $Y-H$ colors for all stars in our sample plus Gl 229b data from Matthews et al. (1996) taken through similar filters in the P200/D-78 camera. Dwarf colors steadily redden from the earliest type stars through at least spectral type L6 stars and brown dwarfs, although they become increasingly bluer from L7 through at least T5. The spectral type labels correspond to $Y-K$ colors; filled symbols indicate increasing color with advancing spectral type (labeled on the left of the sequence), and open symbols indicate decreasing color with advancing spectral type (labeled to the right of the sequence). All objects with $Y-H$ and $Y-K$ colors greater than $3.5 \mathrm{mag}$ are substantially reddened; the derived magnitude and slope of the interstellar reddening vector are indicated by the arrow.

We then employed our $Y-K$ versus $H-K$ diagram to experiment with different values of $A_{Y}$ and find the value that best put the nine reddened stars on the empirically defined color-color line. 2MASS gives only upper limits to the $J$ magnitudes for two of the nine stars, implying lower limit values of $A_{V}$ derived from the $J-H$ versus $H-K_{s}$ diagram; these stars were given little weight in the $A_{Y}$ determination. The relation $A_{Y}=(0.38 \pm 0.03) A_{V}$ was found to best bring the ensemble of stars onto the color sequence defined by unreddened stars. The derived value of total extinction at the $Y$ band relative to the $V$ band is within the rough expectations based on interpolation between adjacent atmospheric windows, given $A_{J}=$ $0.265 A_{V}$ and $A_{I}=0.617 A_{V}$. For comparison, $A_{z(\text { Gunn })}=$ $0.472 A_{V}$ and $A_{z \text { (Sloan })}=0.453 A_{V}$ have been quoted in the literature (Schlegel, Finkbeiner, \& Davis 1998). No information is available to the authors regarding $A_{Z(\mathrm{UFTI})}$.

\section{DISCUSSION}

Soon after incorporation of the $H$ band into standard filter sets, it was shown by Bahng (1969) that the colors of GKM stars differ from blackbody expectations in the near-infrared.
The excursions from monotonic reddening with advancing spectral type are largely due to the influence of $\mathrm{H}^{-}$opacity in the $H$ band. Deviation from blackbody colors is apparent in the now-familiar $J-H$ versus $H-K$ diagram in the growth of $J-H$ relative to $H-K$ in a manner that is "faster" than blackbody from spectral types $\mathrm{G}-\mathrm{K}$, and then in the peak and blueward turnover of $J-H$ near spectral type $\mathrm{K} 7$ as $H-K$ continues to slowly get redder. After spectral type M4, $J-H$ again grows redder with increasing $H-K$ through $\sim \mathrm{L} 6$.

In our data we see that colors involving $Y$ also steadily redden out to $Y-J \approx 1, Y-H \approx 2.5$, and $Y-K \approx 3$ mag at spectral type L6 V. Then, both $Y-H$ and $Y-K$ colors turn sharply blueward in the L6-L8 $\mathrm{V}$ temperature range with later T-type objects having colors similar to those of warmer $\mathrm{L}$ and $\mathrm{M}$ stars. $Y-J$ colors turn only softly blueward, remaining relatively constant at $1.0 \pm 0.15$ mag from early-L through late-T-type dwarfs.

Cool M- and L-type objects initially become redder with advancing spectral type because of increased opacity sources at the shorter $Y$ band, combined with a shift in the peak of the Planck function of $Y$ into $J$. When $\mathrm{CH}_{4}$ opacity begins to contribute substantially in the T dwarfs, the $H$ and $K$ fluxes decrease, sending the colors bluer. This bluing behavior is consistent with that exhibited in $J-H$ versus $H-K$ diagrams of late-type objects (e.g., Burgasser et al. 2002), where the transition between $\mathrm{L}$ dwarfs and $\mathrm{T}$ dwarfs takes colors from the reddest occupied by stars all the way though the heart of the color-color diagram, past every known astronomical object, to the bluest colors occupied by both hot stars and cool brown dwarfs. In the case of $Y H K$ colors, however, instead of crossing the entire color sequence, the T-dwarf sequence overlaps the only slightly warmer L and M dwarfs (see Figs. 6 and 7). The latest $\mathrm{T}$ dwarfs (>T5) appear to fall more blueward in $Y-K$ than $Y-H$ and hence still may be uniquely distinguishable in this color-color plane. Overall, $Y-J$ appears to be the most robust diagnostic of late-type cool stars and brown dwarfs.

The sharp turnaround in $Y-K$ and $Y-H$ occurring in the $\mathrm{T}$ dwarfs stands in contrast to $\mathrm{T}$ dwarf colors at longer and shorter wavelengths. $K-L^{\prime}$ colors appear to redden out to at least L7, become flat with advancing spectral type from L7 to $\mathrm{T} 1$, and then redden again from T1 to at least T6 (Stephens et al. 2001; Leggett et al. 2002). However, hampering the utility of this color diagnostic is that $L$-band data on stellar/substellar photospheres are hard to come by because of high thermal backgrounds and resulting low sensitivity. $I-z$ colors have been studied through only the L spectral types, with Steele \& Howells (2000) claiming that $I-z$ colors redden through the $\mathrm{M}$ and early-L range, flatten from L1 to L5, and then redden again toward even later spectral types. However, $I$-band photometry is not readily obtainable for many of the nearby $\mathrm{L}$ and T objects because of source faintness $\left(M_{I}=15\right.$ mag at M9 but is greater than $18 \mathrm{mag}$ by L7). We thus advocate the use of $Y-K, Y-H$, and $Y-J$ colors for identifying very cool objects.

The downside of the $Y-K$ versus $Y-H$ diagram is the effect of extinction, which drives stars nearly directly along the dwarf 

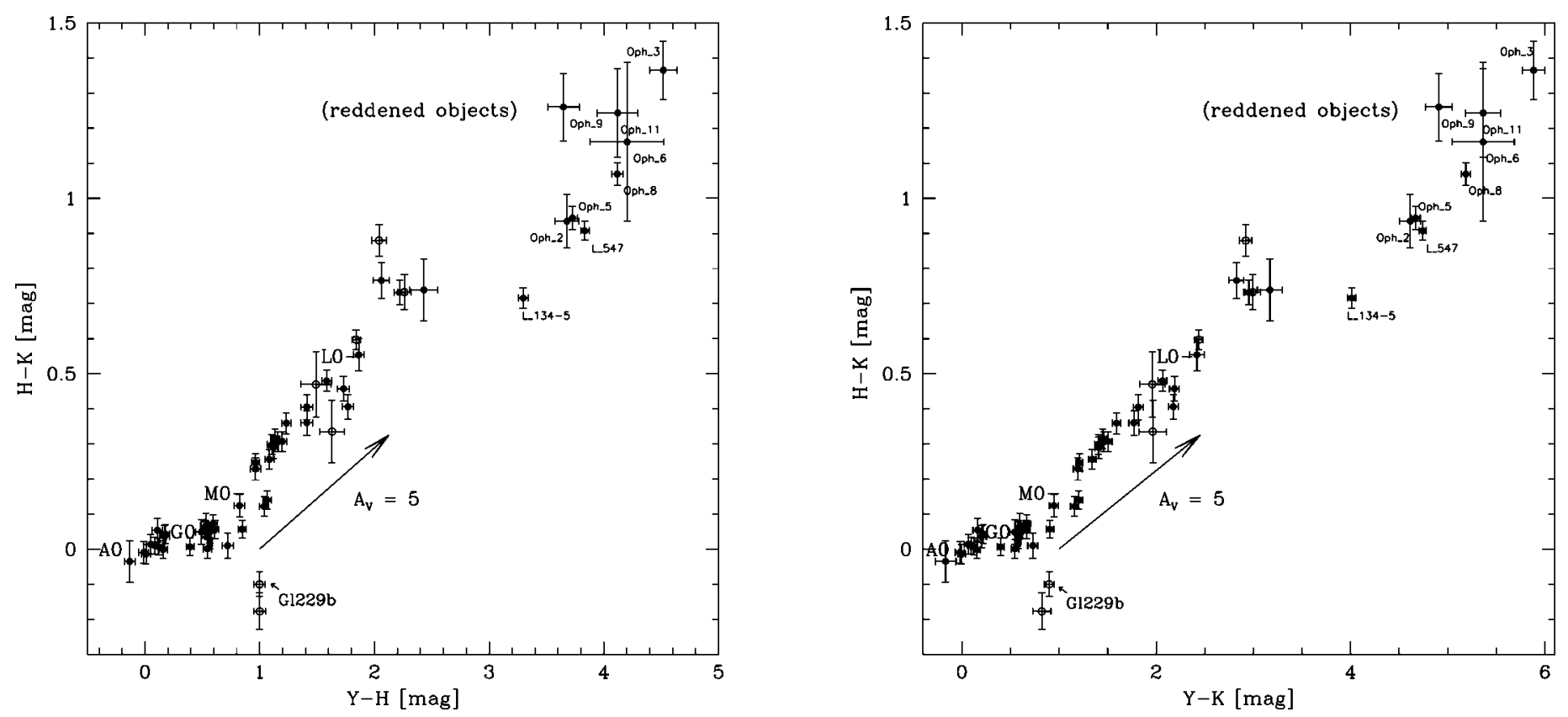

FIG. 7. $-H-K$ colors vs. $Y-H$ (left) and $Y-K$ (right) colors, similar in other ways to Fig. 6 . The centers of the spectral type labels correspond to $H-K$ colors. $H-K$ colors are relatively flat through spectral type $\mathrm{K} 7$ and then redden to $\sim 0.75$ by L6, flatten to $\mathrm{L} 8$, and turn blueward at later types. $Y-K$ and $Y-H$ colors redden more rapidly than $H-K$, though all $Y H K$ colors turn blueward by late $\mathrm{L}$ and into the $\mathrm{T}$ spectral range.

locus. In other color-color planes such as $I-Y$ or $Y-J$ versus $Y-K$ and $J-K$, however, there is more leverage of extinction versus the stellar/substellar color sequence.

\section{CONCLUSIONS}

We present the first exploration of the colors of normal dwarf stars (and some brown dwarfs) spanning spectral types sdO through T5 $\mathrm{V}$ using the $Y$ band at $1.035 \mu \mathrm{m}$. The behavior of the Earth's atmosphere is relatively clean in this window of near unit transparency and few emission lines, especially compared to the other near-infrared $(J, H$, and $K)$ windows. Atmospheric extinction at the $Y$ band is less than 0.05 mag per air mass. The relationship between interstellar extinction in the $Y$ band and that at the $V$ band is $A_{Y}=(0.38 \pm 0.03) A_{V}$.

Our photometric results, including errors, appear in Table 2. Although for most objects only one measurement was taken, photometric variability should not be an issue since our stars are mostly accepted photometric standards in either the optical or the near-infrared. The exception to this are the late-type dwarfs (late $\mathrm{M}, \mathrm{L}$, and $\mathrm{T}$ spectral types) for which smallamplitude near-infrared variability has been reported in some objects (e.g., Bailer-Jones \& Mundt 2001).

The large range in color exhibited by low-mass stars and brown dwarfs makes colors involving the $Y$ band particularly diagnostic of these extremely cool objects. The $I-Y, Y-J$, $Y-H$, and $Y-K$ colors all grow redder with increasing spectral type through at least L6 V. At later spectral types, YJHK colors all saturate and turn blueward to somewhat differing degrees, the result of the strong peak in the spectral energy distributions of extremely cool objects in the 1.1-1.3 $\mu \mathrm{m}$ region. This bluing is caused by $\mathrm{H}_{2} \mathrm{O}, \mathrm{CH}_{4}$, and collision-induced $\mathrm{H}_{2}$ opacity suppressing first the $K$-band and then the $H$-band flux as temperatures grow cooler (e.g., Marley et al. 2002). Infrared spectroscopy of Gl 229b and Gl 570d, the coolest known T dwarfs, appear to confirm these statements for most of the $\mathrm{T}$ spectral type range.

Colors involving the $Y, J, H$, and $M$ bands can uniquely identify extremely cool brown dwarfs and so-called planetarymass objects apart from other astronomical targets and should form the basis for deep surveys of field and cluster samples in the future.

We acknowledge with appreciation Adam Burgasser for obtaining P60/IRC (" $X$ ") $Y$-band data for us of several T dwarfs. L. A. H. thanks John Carpenter for allowing her to talk him into observing with the Keck/NIRC (" $Z$ ") $Y$-band filter during data acquisition for our $H / K$ study of Orion Nebula Cluster faint stars and brown dwarfs. This publication makes use of data products from the Two Micron All Sky Survey (2MASS) and has benefited from information provided by the SIMBAD database. 


\section{REFERENCES}

Bahng, J. 1969, MNRAS, 143, 73

Bailer-Jones, C. A. L., \& Mundt, R. 2001, A\&A, 367, 218

Barsony, M., Kenyon, S. J., Lada, E. A., \& Teuben, P. J. 1997, ApJS, 112, 109

Bessell, M. S., \& Brett, J. M. 1988, PASP, 100, 1134

Burgasser, A. J., et al. 2002, ApJ, 564, 421

Burrows, A., Hubbard, W. B., Lunine, J. I., \& Liebert, J. 2001, Rev. Mod. Phys., 73, 719

Burrows, A., et al. 1997, ApJ, 491, 856

Cohen, J. G., Persson, S. E., Elias, J. H., \& Frogel, J. A. 1981, ApJ, 249,481

Fukugita, M., Ichikawa, T., Gunn, J. E., Doi, M., Shimasaku, K., \& Schneider, D. P. 1996, AJ, 111, 1748

Geballe, T. R., et al. 2002, ApJ, 564, 466

Hawarden, T. G., Leggett, S. K., Letawsky, M. B., Ballantyne, D. R., \& Casali, M. M. 2001, MNRAS, 325, 563

Hawley, S. L., Gizis, J. E., \& Reid, I. N. 1996, AJ, 112, 2799

Hillenbrand, L. A., \& Carpenter, J. M. 2000, ApJ, 540, 236

Johnson, H. L. 1966, ARA\&A, 4, 193

Kirkpatrick, J. D., Henry, T. J., \& McCarthy, D. W. 1991, ApJS, 77, 417

Kirkpatrick, J. D., Henry, T. J., \& Simons, D. A. 1995, AJ, 109, 797

Kirkpatrick, J. D., et al. 1999, ApJ, 519, 802 2000, AJ, 120, 447

Landolt, A. U. 1992, AJ, 104, 340

Lasker, B. M., et al. 1988, ApJS, 68, 1

Leggett, S. K. 1992, ApJS, 82, 351

Leggett, S. K., Allard, F., Dahn, C., Hauschildt, P. H., Kerr, T. H., \& Rayner, J. 2000a, ApJ, 535, 965

Leggett, S. K., Allard, F., \& Hauschildt, P. H. 1998, ApJ, 509, 836
Leggett, S. K., et al. 2000b, ApJ, 536, L35

2002, ApJ, 564, 452

Manduca, A., \& Bell, R. A. 1979, PASP, 91, 848

Marley, M. S., Saumon, D., Guillot, T., Freedman, R. S., Hubbard, W. B., Burrows, A., \& Lunine, J. I. 1996, Science, 272, 1919

Marley, M. S., Seager, S., Saumon, D., Lodders, K., Ackerman, A. S., \& Freedman, R. 2002, ApJ, 568, 335

Matthews, K., Nakajima, T., Kulkarni, S. R., \& Oppenheimer, B. R. 1996, AJ, 112, 1678

Matthews, K., \& Soifer, B. T. 1994, in Infrared Astronomy with Arrays: The Next Generation, ed. I. McLean (Dordrecht: Kluwer), 239

Murphy, D. C., Persson, S. E., Pahre, M. A., Sivaramakrishnan, A. M., \& Djorgovski, S. G. 1995, PASP, 107, 1234

Nakajima, T., Oppenheimer, B. R., Kulkarni, S. R., Golimowski, D. A., Matthews, K., \& Durrance, S. T. 1995, Nature, 378, 463

Persson, E., Murphy, D. C., Krzeminski, W., Roth, M., \& Rieke, M. J. 1998, AJ, 116, 2475

Reid, I. N., Hawley, S. L., \& Gizis, J. E. 1995, AJ, 110, 1838

Schlegel, D. J., Finkbeiner, D. P., \& Davis, M. 1998, ApJ, 500, 525

Selby, J. E. A., \& McClatchey, R. A. 1975, Atmospheric Transmittance From 0.25 to $28.5 \mu \mathrm{m}$ : Computer Code LOWTRAN 3 (May 7; Cambridge: Air Force Cambridge Research Laboratories)

Steele, I. A., \& Howells, L. 2000, MNRAS, 313, L43

Stephens, D. C., Marley, M. S., Noll, K. S., \& Chanover, N. 2001, ApJ, 556, L97

Tinney, C. G. 1993, ApJ, 414, 279

Tokunaga, A. T., Simons, D. A., \& Vacca, W. D. 2002, PASP, 114, 180

Note added in proof.-A recent preprint by C. Dahn et al. (2002) reports $V R I z^{*} / J H K$ photometry for seven cool stars and brown dwarfs in common with our study. Examination of the $z^{*}-Y$ color shows valves generally, although nonmonotonically, increasing from $1.5 \mathrm{mag}$ at spectral type L1 V to $2.4 \mathrm{mag}$ at spectral type T6.5 V. 